Commun. math. Phys. 3, 53-74 (1966)

\title{
Analytic Continuation of Group Representations II*
}

\author{
Robert HermanN \\ Applied Mathematics Division, Argonne National Laboratory
}

Received April 22, 1966

\begin{abstract}
The paper deals with the general background connecting the ideas of analytic continuation and contraction of Lie algebras and their representations. A connection is also established between the Kodaira-Spencer deformation theory and the theory of cohomology of Lie algebras.
\end{abstract}

\section{Introduction}

In this paper, we follow up some relations discovered in [1] between the contraction of a Lie algebra and the various "analytic continuations" possible for its skew-Hermitian representations. Consider the Lie algebra of $S L(2, R)$. One contraction leads to the group of rigid motions in the plane. Conversely, through an "expansion" process (using the "GellMann formula") a representation of the latter group gives a one parameter family of representations of $S L(2, R)$, which is just the familiar "continuous series". Another contraction leads to the Heisenberg Lie algebra, i.e. to that generated by the Heisenberg commutation relations $[p, q]=1,0=[1, p]=[1, q]$. Dothas has pointed out that there is an opposite "expansion" process. (It will be presented in this paper.) It has a new feature: Although the formulas depend continuously on the parameter, only for discrete values of this parameter do the formulas give genuine non-singular operators. The corresponding series of representations of $S L(2, R)$ is the "discrete series", while the associated "physics" is that of the one dimensional harmonic oscillator.

We hope that ultimately these facts will be generalized to the other semisimple groups. The intuitive geometric picture at which we are aiming is that of the equivalence classes of unitary representations of a given Lie group forming a non-compact, finite dimensional space, with the representations of the various contractions of the given group lying on the boundary as "points at infinity".

In working on this program, it will be useful to have the general ideas of "deformation" of Lie algebras and their representations at hand. This fits into a much more general pattern of mathematical thought, first created by K. Kodatra and D. C. SPENCER, and carried further by many others, e.g., E. Calabi, M. Gerstenhaber, P. Griffiths, M.

\footnotetext{
* Supported by the Office of Naval Research, NONR 3656(09).
} 
Kuranishi, A. NiJenhuis and R. Richardson, in various algebraic and geometric directions. (A review paper by NIJENHUIS and RICHARDson [3] is most closely relevant for our work and we refer there for further references.) One interesting feature of the theory is that the idea of cohomology of Lie algebras appears very naturally. Since the available mathematical literature on this is rather impenetrable to the nonspecialist, we will give in this paper an independent treatment of this theory, together with some indications how it applies to the theory of deformations and show that the deformation theory is related to the basic problems in group representation theory.

This work owes much to conversations with N. BuRgoyne, Y. Dothan, M. Gell-Mann and D. C. Spencerr, and I would like to thank them.

\section{Analytic continuations of Lie algebras and their representations}

Let $\mathrm{G}$ be a Lie algebra over the reals numbers. Let us denote elements of $G$ by such letters as $X, Y$, etc., and denote the given Lie algebra bracket by $[X, Y]$. An analytic continuation or deformation of this Lie algebra is a one-parameter ${ }^{1}$ family of Lie algebra structures on the same underlying vector spaces as $G$, reducing to the given one at $\lambda=0$. We denote the bracket in the Lie algebra parameterized by $\lambda$ as:

$$
[X, Y]_{\lambda} \text {. }
$$

A Lie algebra structure $(X, Y) \rightarrow[X, Y]_{\infty}$ is the limit of the deformation if:

$$
\lim _{\lambda \rightarrow \infty}[X, Y]_{\lambda}=[X, Y]_{\infty} \text { for } X, Y \in \mathbf{G} .
$$

(We will not try to keep track of the subtleties involved in the topology if $\mathbf{G}$ is infinite dimensional: Let us say that we are working explicitly in the finite dimensional case but keep in mind that some of the ideas should apply to the infinite dimensional case also.)

Now, we want to describe how deformation of representations is defined when such a deformation of Lie algebras is given. Let $\mathbf{L}$ be another Lie algebra, and let $\varphi$ be a homomorphism of the original Lie algebra structure on $\mathbf{G}$ into $\mathbf{L}$, i.e.

$$
\varphi([X, Y])=[\varphi(X), \varphi(Y)] \text { for } X, Y \in \mathbf{G} .
$$

A deformation on analytic continuation of $\varphi$ is a one parameter family $\lambda \rightarrow \varphi_{\lambda}$ of linear maps: $\mathbf{G} \rightarrow \mathbf{L}$, such that, for each $\lambda, \varphi_{\lambda}$ is a homomorphism of the $\lambda$-th Lie algebra structure on $G$ into that on $\mathbf{L}$, i.e.

$$
\varphi_{\lambda}\left([X, Y]_{\lambda}\right)=\left[\varphi_{\lambda}(X), \varphi_{\lambda}(Y)\right] \text { for } X, Y \in \mathbb{G},
$$

which reduces to $\varphi$ at $\lambda=0$.

1 For simplicity in stating the ideas, we are considering deformations varying smoothly (i.e. with all necessary derivatives) with a real parameter $\lambda$. Of course, many of the ideas carry over to more general parameter spaces. 
The limit map $\varphi_{\infty}$ is defined as:

$$
\varphi_{\infty}(X)=\lim _{\lambda \rightarrow \infty} \varphi_{\lambda}(X) \text { for } X \in \mathbf{G} .
$$

It is clearly a homomorphism of the $[,]_{\infty}$-Lie algebra structure defined by (2.1) into $\mathbf{L}$.

The Inonu-Segal-Wigner ideas of "contraction" of Lie algebras and representations is a special case: Suppose that the $[,]_{\lambda}$-Lie algebra is isomorphic to the original one, i.e. there is, for each $\lambda$, an invertible linear $\operatorname{map} A_{\lambda}: \mathbf{G} \rightarrow \mathbf{G}$ such that

Then

$$
[X, Y]_{\lambda}=A_{\lambda}^{-1}\left[A_{\lambda} X, A_{\lambda} Y\right] .
$$

Let $\varphi_{\lambda}^{\prime}=\varphi_{\lambda} A_{\lambda}^{-1}$. Then

$$
[X, Y]_{\infty}=\lim _{\lambda \rightarrow \infty} A_{\lambda}^{-1}\left[A_{\lambda} X, A_{\lambda} Y\right]
$$

$$
\begin{aligned}
\varphi_{\lambda}^{\prime}[X, Y] & =\varphi_{\lambda} A_{\lambda}^{-1}[X, Y] \\
& =\varphi_{\lambda} A_{\lambda}^{-1}\left[A_{\lambda} A_{\lambda}^{-1} X, A_{\lambda} A_{\lambda}^{-1} Y\right] \\
& =\varphi_{\lambda}\left(\left[A_{\lambda}^{-1} X, A_{\lambda}^{-1} Y\right]_{\lambda}\right) \\
& =\left[\varphi^{\prime}(X), \varphi^{\prime}(Y)\right],
\end{aligned}
$$

i.e. $\lambda \rightarrow \varphi_{\lambda}^{\prime}$ is a one-parameter family of homomorphisms of the original Lie algebra structure on $\mathbf{G}$ into $\mathbf{L}$. Conversely if the deformation $\varphi_{\lambda}^{\prime}$ is given as a one-parameter family of homomorphisms of the given Lie algebra structure on $\mathbf{G}$, defining

$$
\varphi_{\lambda}=\varphi_{\lambda}^{\prime} A_{\lambda}
$$

provides a one-parameter family of homomorphisms of the $\lambda$-th structure on $\mathrm{G}$. Thus, we may consider $\varphi_{\infty}$ defined as $\lim _{\lambda \rightarrow \infty} \varphi_{\lambda}^{\prime} A_{\lambda}$ : It may converge, even though the limit Lie algebra $[,]_{\infty}$ does not: Thus, one might be able at least in principle, to "contract" a representation of a Lie algebra without being able to contract the Lie algebra itself. (However, I know of no such examples.)

Let us look at an example: Take $\mathbf{G}$, with its "original" Lie algebra structure, as the Lie algebra of $S L(2, R)$. It has a basis given by $X, Y, Z$,

$$
\begin{aligned}
{[Z, X] } & =Y ;[Z, Y]=-X ; \\
{[X, Y] } & =-Z .
\end{aligned}
$$

One contraction leads to the Lie algebra of the group of rigid motions in the plane:

$$
\begin{aligned}
A_{\lambda} Z & =Z ; \\
A_{\lambda} X & =X / \lambda ; A_{\lambda} Y=Y / \lambda .
\end{aligned}
$$


Then,

$$
\begin{aligned}
& {[X, Z]_{\infty}=Y ;[Z, Y]_{\infty}=-X ;} \\
& {[X, Y]_{\infty}=0 .}
\end{aligned}
$$

To keep notation from getting unduly complicated, let us denote this Lie algebra in the following way: It is given by elements $Z^{\prime}, X^{\prime}, Y^{\prime}$ satisfying:

$$
\begin{aligned}
& {\left[Z^{\prime}, X^{\prime}\right]=Y^{\prime} ;\left[Z^{\prime}, Y^{\prime}\right]=-X^{\prime} ;} \\
& {\left[X^{\prime}, Y^{\prime}\right]=0 .}
\end{aligned}
$$

Let $\mathbf{L}$ be the "complete" universal enveloping algebra of the Lie algebra defined by (2.4), i.e. the Lie algebra formed by taking formal power series in its elements. Then, the "Gell-Mann formula" [1] asserts that the following formulas define a one-parameter family of homomorphisms of $\mathbf{G} \rightarrow \mathbf{L}:$

$$
\begin{aligned}
& \varphi_{\lambda}^{\prime}(X)=\frac{1}{2 \delta}\left[Z^{\prime 2}, X^{\prime}\right]+\lambda X^{\prime} \\
& \varphi_{\lambda}^{\prime}(Y)=\frac{1}{2 \delta}\left[Z^{\prime 2}, Y^{\prime}\right]+\lambda Y^{\prime} \\
& \varphi_{\lambda}(Z)=Z^{\prime} ; \delta^{2}=X^{\prime 2}+Y^{\prime 2} .
\end{aligned}
$$

Notice now that

$$
\varphi_{\lambda}=\varphi_{\lambda}^{\prime} A_{\lambda}
$$

converges as $\lambda \rightarrow \infty$ to the map

$$
X \rightarrow X^{\prime} ; Y \rightarrow Y^{\prime} ; Z \rightarrow Z^{\prime}
$$

which sets up the isomorphism between $[,]_{\infty}$ and the Lie algebra defined by (2.4).

Another contraction of interest is that where $G$ contracts into the Heisenberg Lie algebra:

Then,

$$
A_{\lambda}(Z)=Z / \lambda ; A_{\lambda}(X)=X / \sqrt{\lambda} ; A_{\lambda}(Y)=Y / \sqrt{\lambda}
$$

$$
\begin{aligned}
& {[Z, X]_{\infty}=0=[Z, Y]_{\infty}} \\
& {[X, Y]_{\infty}=-Z}
\end{aligned}
$$

which are the structure relations of the Heisenberg algebra (with the identifications $Z \rightarrow 1 ; Y \rightarrow p ; X \rightarrow q)$.

Now, let $\mathbf{L}$ be the Lie algebra of functions $f(p, q)$ of two real variables $p$ and $q$, with the Lie algebra structure given by the Poisson bracket:

$$
\{f, g\}=\frac{\partial f}{\partial p} \frac{\partial g}{\partial q}-\frac{\partial f}{\partial q} \frac{\partial g}{\partial p} \text {. }
$$


Now, consider:

$$
\begin{aligned}
\varphi_{\lambda}^{\prime}(Z) & =H+\lambda \\
\varphi_{\lambda}^{\prime}(X) & =\sqrt{H / 2+\lambda} q \\
\varphi_{\lambda}^{\prime}(Y) & =\sqrt{H / 2+\lambda} p, H=\frac{1}{2}\left(p^{2}+q^{2}\right) .
\end{aligned}
$$

It is readily checked that, for every $\lambda$, this is a homomorphism of $\mathbf{G}$ into L. Obviously, $\varphi_{\lambda}^{\prime} A$ converges as $\lambda \rightarrow \infty$ to the isomorphism between $[,]_{\infty}$ and the Heisenberg algebra, generated by $1, p$ and $q$.

I owe this construction to Y. Dothan. (He has also generalized it to $S U(n, 1))$. It is clearly the analog of the "Gell-Mann formula" for this particular contraction of $\mathbf{G}$, enabling one to "expand" a representation of the contracted algebra to a one-parameter family of representations of the original Lie algebra which has automatically the correct limiting properties as the parameter goes to infinity. This example leads one to conjecture that there are many such formulas yet to be discovered by considering the multitude of possible contractions of a given semisimple Lie algebra.

\section{Cohomology of Lie algebras}

We have just seen that there is great interest from the point of view of representation theory in the problem of knowing in how many different ways it is possible to "deform" a given Lie algebra or Lie algebra homomorphism. The Kodaira-Spencer theory, mentioned in the introduction, is designed to do precisely this. There is one feature to our problem that is new : their theory is designed to study the deformation of structures in a small neighborhood of the origin of the deformation parameter, while we are also interested in the limiting behavior as the deformation parameter goes to infinity. Since there do not as yet seem to be any systematic techniques for studying this latter problem, it seems natural to ask what the existing ideas have to offer. We will give a brief review of this here, presenting a different approach, using the idea of "Lie derivative". (In the background, is an approach to differential geometry presented in [2].)

Let $\mathbf{G}$ be a Lie algebra, as before, and let $V$ be a vector space. Suppose $\varphi$ is a linear representation of $\mathbf{G}$ to operators on $V$, i.e. to each $X \in \mathbf{G}$ is assigned a linear transformation $\varphi(X): V \rightarrow V$, such that

$$
\varphi([X, Y])=\varphi(X) \varphi(Y)-\varphi(Y) \varphi(X) \text { for } X, Y \in \mathbf{G} .
$$

An $r$-cochain, usually denoted by $\omega$, will be a multilinear, skew-symmetric function $\left(X_{1}, \ldots, X_{r}\right) \rightarrow \omega\left(X_{1}, \ldots, X_{r}\right)$ from $r$-tuples of $G$ to elements of $V$. We can symbolize this by:

$$
\omega: \mathbf{G} \wedge \cdots \wedge \mathbf{G} \rightarrow V .
$$


The set of all these cochains forms a vector space, that we will denote by $C^{r}(\varphi)$. We will first define two operations between elements of $G$ and $r$-cochains, the inner product and the Lie derivative.

a) If $\left.X \in \mathbf{G}, \omega \in C^{r}(\varphi),(X\rfloor \omega\right)$, the inner product of $\omega$ and $X$, is an element of $C^{r-1}(\varphi)$ which assigns to $\left(X_{1}, \ldots, X_{r-1}\right)$ the element $\omega\left(X, X_{1}, \ldots, X_{r-1}\right)$, i.e. $\left.(X\rfloor \omega\right)\left(X_{1}, \ldots, X_{r-1}\right)=\omega\left(X, X_{1}, \ldots, X_{r-1}\right)$.

b) For $X \in G, \omega \in C^{r}(\varphi), X(\omega)$, the Lie derivative of $\omega$ by $X$, is an element of $C^{r}(\varphi)$ defined by:

$$
\begin{aligned}
X(\omega)\left(X_{1}, \ldots, X_{r}\right)=\varphi(X)\left(\omega\left(X_{1}, \ldots, X_{r}\right)\right)- & \\
& -\omega\left(\left[X, X_{1}\right], X_{2}, \ldots, X_{r}\right)-\cdots-\omega\left(X_{1}, \ldots,\left[X, X_{r}\right]\right) \\
& \text { for } X_{1}, \ldots, X_{r} \in \mathbf{G} .
\end{aligned}
$$

(It can be readily verified that this formula really depends skew-symmetrically on $X_{1}, \ldots, X_{r}$.) A special convention is necessary for $r=0: C^{0}(\varphi)$ will be defined to be $V$ itself. If $v \in C^{0}(\varphi)=V$, then

$$
\begin{aligned}
X \downarrow v & =0 \\
X(v) & =\varphi(X)(v) .
\end{aligned}
$$

Now, we will define a linear mapping

$$
d: C^{r}(\varphi) \rightarrow C^{r+1}(\varphi)
$$

by induction on $r$. ( $d$ is called the exterior derivative.) If $r=0, v \in C^{0}(\varphi)$, $d v \in C^{1}(\varphi)$. As definition,

$$
d v(X)=\varphi(X)(v) \text { for } X \in \mathbf{G} .
$$

Suppose now that $d$ is defined for all degrees less than $r$, and that $\omega \in C^{r}(\varphi)$. As definition,

$\left.d \omega\left(X_{1}, \ldots, X_{r+1}\right)=X_{1}(\omega)\left(X_{2}, \ldots, X_{r+1}\right)-d\left(X_{1}\right\rfloor \omega\right)\left(X_{2}, \ldots, X_{r+1}\right)$ for $X_{1}, \ldots, X_{r} \in G$.

Thus, the three basic operations are related as follows:

$$
X(\omega)=X \downarrow d \omega+d(X\rfloor \omega) \text { for } X \in \mathbf{G}, \omega \in C^{r}(\varphi) .
$$

Lemma 3.1. For $X, Y \in \mathbb{G}, \omega \in C^{r}(F, \varphi)$,

Proof.

$$
X(Y\rfloor \omega)=[X, Y]\rfloor \omega+Y\rfloor X(\omega) .
$$

$$
\begin{aligned}
& X(Y \perp \omega)\left(X_{1}, \ldots, X_{r-1}\right)=\varphi(X)\left(\omega\left(Y, X_{1}, \ldots, X_{r-1}\right)\right)- \\
&-\omega\left(Y,\left[X, X_{1}\right], \ldots, X_{r-1}\right)-\cdots-\omega\left(Y, X_{1}, \ldots,\left[Y, X_{r-1}\right]\right) \\
& {[X, Y]\rfloor \omega\left(X_{1}, \ldots, X_{r-1}\right)=} \omega\left([X, Y], X_{1}, \ldots, X_{r-1}\right) \\
& Y \perp X(\omega)\left(X_{1}, \ldots, X_{r-1}\right)= X(\omega)\left(Y, X_{1}, \ldots, X_{r-1}\right) \\
&= \varphi(X)\left(\omega\left(Y, X_{1}, \ldots, X_{r-1}\right)\right)- \\
&-\omega\left([X, Y], X_{1}, \ldots, X_{r-1}\right)-\cdots \\
& \cdots-\omega\left(Y, X_{1}, \ldots,\left[X, X_{r-1}\right]\right) .
\end{aligned}
$$

This shows explicitly that the formula is true. 
Lemma 3.2. $d \omega$ so defined depends skew-symmetrically on

$$
X_{1}, \ldots, X_{r+1} \text {. }
$$

Proof. Proceed by induction on $r$. The induction hypotheses shows that $d \omega$ depends skew-symmetrically on $X_{2}, \ldots, X_{r+1}$. We must verify that it changes sign under interchange of $X_{1}$ and $X_{2}$. But

$$
\begin{aligned}
\left.d\left(\left(X_{1}\right\lrcorner \omega\right)\right)\left(X_{2}, \ldots, X_{r+1}\right)= & X_{2}((X\rfloor \omega)\left(X_{3}, \ldots, X_{r+1}\right) \\
& \left.\left.-d\left(X_{2}\right\rfloor X_{1}\right\lrcorner \omega\right)\left(X_{3}, \ldots, X_{r+1}\right) .
\end{aligned}
$$

By Lemma 3.1,

$$
\begin{aligned}
\left.X_{2}\left(\left(X_{1}\right\rfloor \omega\right)\right)\left(X_{3}, \ldots, X_{r-1}\right)= & \left.\left(\left[X_{2}, X_{1}\right]\right\lrcorner \omega\right)\left(X_{3}, \ldots, X_{r-1}\right)+ \\
& \left.+X_{1}\right\lrcorner X_{2}(\omega)\left(X_{3}, \ldots, X_{r-1}\right) \\
= & \left.\left(\left[X_{2}, X_{1}\right]\right\rfloor \omega\right)\left(X_{3}, \ldots, X_{r-1}\right)+ \\
& +X_{2}(\omega)\left(X_{1}, X_{3}, \ldots, X_{r-1}\right) .
\end{aligned}
$$

This shows that $d \omega$ depends skew-symmetrically on $X_{1}$ and $X_{2}$.

Lemma 3.3. For $X, Y \in \mathbf{G}, \omega \in C^{r}(\varphi)$,

$$
[X, Y](\omega)=X(Y(\omega))-Y(X(\omega)) .
$$

Proof. Proceed by induction on $r$. Let $Z \in \mathbf{G}$.

$$
\begin{aligned}
Z \perp[X, Y](\omega)= & {[X, Y](Z\rfloor \omega)-[[X, Y], Z]\rfloor \omega } \\
Z\lrcorner X Y(\omega)= & X(Z\rfloor Y(\omega))-[X, Z]\rfloor Y(\omega) \\
= & X(Y(Z\rfloor \omega)-[Y, Z]\rfloor \omega)- \\
& -Y([X, Z]\rfloor \omega)+[Y,[X, Z]]\rfloor \omega .
\end{aligned}
$$

By induction hypotheses,

Thus

$$
X Y(Z\rfloor \omega)-Y X(Z\rfloor \omega)=[X, Y](Z\rfloor \omega) .
$$

$$
\begin{aligned}
&Z\rfloor([X, Y](\omega)-X Y(\omega)+Y X(\omega)) \\
&=[X, Y](Z \omega)-[[X, Y], Z]\rfloor \omega- \\
&-[X, Y](Z \omega)+X([Y, Z]\rfloor \omega+ \\
&+Y([X, A]\lrcorner \omega)-[Y,[X, Z]]\rfloor \omega- \\
&-Y([X, Z] \omega)-X([Y, Z] \omega)+ \\
&+[X,[Y, Z]]\rfloor \omega,
\end{aligned}
$$

which is zero by virtue of the Jacobi identity for $G$. Since $Z$ is arbitrary, the lemma is proved. (An $n$-cochain is zero if and only if its inner product with all elements of $\mathbf{G}$ is zero.) 
Lemma 3.4. $X(d \omega)=d(X(\omega))$ for $\omega \in C^{r}(\mathbf{G}(\varphi)), X \in \mathbf{G}$.

Proof. Again, proof is by induction on $r$. Let $Y$ be an element of $\mathbf{G}$.

$$
\begin{aligned}
Y\lrcorner X(d \omega)= & -[X, Y]\rfloor d \omega+X(Y\rfloor d \omega) \\
= & -[X, Y]\rfloor d \omega+X(Y(\omega)-d(Y\rfloor \omega)) \\
= & -([X, Y](\omega)-d([X, Y]\rfloor \omega))+ \\
& +X Y(\omega)-X d(Y\rfloor \omega) \\
= & Y X(\omega)+d([X, Y]\rfloor \omega)-d X(Y\rfloor \omega) \\
= & Y X(\omega)+d([X, Y]\rfloor \omega)-d([X, Y]\rfloor \omega+Y\rfloor X(\omega) \\
= & Y X(\omega)-Y \perp(\omega) \\
= & Y \perp d(X(\omega)) . \quad \text { q.e.d. }
\end{aligned}
$$

Lemma 3.5. $d d \omega=0$ for $\omega \in C^{r}(G, \varphi)$.

Proof. Again, induction on $r$. For $Y \in G$,

$$
\begin{aligned}
Y\rfloor d d \omega & =Y(d \omega)-d(Y\rfloor d \omega) \\
& =d Y(\omega)-d(Y\rfloor d \omega) \\
& =d(Y\rfloor d \omega+d(Y\rfloor \omega)-d(Y\rfloor d \omega)) \\
& =d d(Y\rfloor \omega)=0, \text { by induction hypotheses. q.e.d. }
\end{aligned}
$$

These lemmas provide us with the basic rules of calculation for the three operations of inner product, Lie derivative and exterior derivative.

Let us calculate, for example, $d \omega$ for $\omega \in C^{r}(G, \varphi), r=1,2$. $r=1$.

$$
\begin{aligned}
& r=1 . \begin{aligned}
d \omega(X, Y) & =(X\rfloor d \omega)(Y) \\
& =X(\omega)(Y)-d(X\rfloor \omega)(Y) \\
& =\varphi(X)(\omega(Y))-\omega([X, Y])-\varphi(Y)(\omega(X)) .
\end{aligned} \\
&r=2 . d \omega(X, Y, Z)=(X\rfloor d \omega)(Y, Z)
\end{aligned}
$$

$$
\begin{aligned}
= & X(\omega)(Y, Z)-d(X\rfloor \omega)(Y, Z) \\
= & \varphi(X)(\omega(Y, Z))-\omega([X, Y], Z)-\omega(Y,[X, Z])- \\
& -\varphi(Y)((X\rfloor \omega)(Z))+\varphi(Z)((X\rfloor \omega)(Y))+ \\
& +(X\rfloor \omega)([Y, Z]) \\
= & \varphi(X)(\omega(Y, Z))-\omega([X, Y], Z)-\omega(Y,[X, Z])- \\
& -\varphi(Y)(\omega(X, Z))+\varphi(Z)(\omega(X, Y))+ \\
& +\omega(X,[Y, Z]) .
\end{aligned}
$$

Lemma 3.5 enables us to define the cohomology groups ${ }^{2} H^{r}(\varphi)$. A cochain $\omega \in C^{r}(\varphi)$ is a cocycle if $d \omega=0$. (Sometimes one also says it is closed.) By Lemma 3.5, if $\omega=d \theta$, for $\theta \in C^{r-1}(\varphi)$, it is a cocycle: One

${ }^{2}$ In this context, they are more naturally thought of as vector spaces. 
says it is a coboundary if such a $\theta$ exists. The set of cocycles form a vector space, called $Z^{r}(\varphi)$. The coboundaries form a subvector space, called $d C^{r-1}(\varphi)$. Now, as definition:

$$
H^{r}(\varphi)=Z^{r}(\varphi) / d C^{r-1}(\varphi),
$$

i.e. $H^{r}(\varphi)$ is the quotient of the cocycles modulo the coboundaries, hence measures the extent to which cocycles fail to be coboundaries.

Notice that the Lie derivative operation $\omega \rightarrow X(\omega)$ defines a representation of $\mathbf{G}$ by linear transformations on $C^{r}(\varphi)$, which maps $Z^{r}(\varphi)$ and $d C^{r-1}(\varphi)$ into themselves (Lemmas 3.3 and 3.4).

If $\omega \in Z^{r}(\varphi), X \in \mathbf{G}$,

$$
\begin{aligned}
& X(\omega)=d(X\rfloor \omega) \\
& X\left(Z^{r}(\varphi)\right) \subset B^{r}(\varphi),
\end{aligned}
$$

i.e. the quotient linear representation of $\mathrm{G}$ on $H^{r}(\varphi)$ is the trivial representation.

Suppose that there is a subspace $W^{r}(\varphi)$ of $Z^{r}(\varphi)$ such that:

$$
\begin{gathered}
Z^{r}(\varphi)=d C^{r-1}(\varphi) \oplus W^{r}(\varphi) \\
X\left(W^{r}(\varphi)\right) \subset W^{r}(\varphi) \text { for } X \in G .
\end{gathered}
$$

(For example, if $\mathbf{G}$ is finite dimensional and semisimple and if $V$ is finite dimensional, such a subspace exists by the complete reducibility theorem for semisimple Lie algebras.) Then, the action of $\mathbf{G}$ on $W^{r}(\varphi)$, isomorphic to that of $G$ on $H^{r}(\varphi)$. Hence,

$$
X(\omega)=0=d(X \perp \omega) \quad \text { for } \quad \omega \in W^{r}(\varphi) ; X \in \mathbf{G} .
$$

Suppose $r=1$ : Then,

$$
\begin{gathered}
\varphi(Y)(\omega(X))=0 \text { for } X, Y \in \mathbf{G}, \\
\omega([X, Y])=0 .
\end{gathered}
$$

In particular; we have

Theorem 3.6. $H^{1}(\varphi)=0$ if $[\mathbf{G}, \mathbf{G}]=\mathbf{G}$ and if the subspace $W^{1}(\varphi)$ satisfying 3.1 exists.

(For example, $[\mathbf{G}, \mathbf{G}]=\mathbf{G}$ if $\mathbf{G}$ is semisimple).

Now, under the assumption that $H^{1}(\varphi)=0$ and that $W^{2}(\varphi)$ exists, let us examine $H^{2}(\varphi)$. Suppose $\omega \in W^{2}(\varphi)$. Then,

$$
d(X\lrcorner \omega)=0 \text { for } X \in G .
$$

Since $H^{1}(\varphi)=0$, there is a $\theta_{X} \in C^{0}(\varphi)$ with

Then, for $X, Y \in G$,

$$
\left.d \theta_{X}=X\right\rfloor \omega .
$$

$$
d\left(\theta_{X}+\theta_{Y}-\theta_{X+Y}\right)=0 .
$$


Let $v=\theta_{X}+\theta_{Y}-\theta_{X+Y} \in V$. Then,

$$
\varphi(Z)(v)=0 \text { for all } Z \in \mathbf{G} \text {. }
$$

Let us suppose that there are no vectors in $V$ invariant under all of $\varphi(\mathbf{G})$. Then, $v=0$, i.e.

is a linear mapping $\mathbf{G} \rightarrow V$.

$$
X \rightarrow \theta_{X}
$$

Now,

i.e. $d\left(\theta_{[X, Y]}-X\left(\theta_{Y}\right)\right)=0$.

$$
\begin{aligned}
d \theta_{[X, Y]} & =[X, Y]\rfloor \omega \\
& =X(Y\rfloor \omega)-Y\lrcorner X(\omega) \\
& =X\left(d \theta_{Y}\right) \\
& =d\left(X\left(\theta_{Y}\right)\right)
\end{aligned}
$$

Again, since we are assuming that there are no vectors in $V$ invariant under $\varphi(\mathbf{G})$,

$$
\theta_{[X, Y]}=X\left(\theta_{Y}\right)=\varphi(X)\left(\theta_{Y}\right) .
$$

This says that the subspace $\left\{\theta_{X}: X \in \mathbf{G}\right\}$ of $V$ transforms like the adjoint representation of $\mathbf{G}$. This, we have proved:

Theorem 3.7. If $a W^{2}(\varphi)$ satisfying (3.1) exists, if $H^{1}(\varphi)=0$, if $\varphi(\mathbf{G})$ has no invariant vectors, and if $V$ has no subspaces transforming under $\varphi(\mathbf{G})$ like the adjoint representation, then

$$
H^{2}(\varphi)=0 \text {. }
$$

Now, let us consider the case that escapes Theorem 3.7. Suppose that $V=\mathbf{G}$ also, and $\varphi$ is the adjoint representation of $\mathbf{G}$. Thus, $X \rightarrow \theta_{X}$ defines a linear mapping of $G$ into itself. The condition

$$
\theta_{[X, Y]}=\varphi(X) \theta_{Y}
$$

means that this map commutes with the adjoint representation. Suppose first that $G$ is a simple Lie algebra. Then, the adjoint representation is irreducible, hence $X \rightarrow \theta_{Y}$ must be a multiple of the identity, i.e.

for some scalar $c$.

$$
\theta_{X}=c X \text {, }
$$

Thus,

$$
\begin{aligned}
\omega(X, Y) & =(X\rfloor \omega)(Y)=d\left(\theta_{X}\right)(Y) \\
& =\varphi(Y)\left(\theta_{X}\right)=c[Y, X] .
\end{aligned}
$$

Let $\alpha \in C^{\mathbf{1}}(\varphi)$ be $c$ times the identity map:

i.e.

$$
\begin{aligned}
d \alpha(X, Y) & =\varphi(X)(\alpha(Y))-\varphi(Y)(\alpha(X))-([X, Y]) \\
& =c[X, Y]-c[Y, X]-c[X, Y] \\
& =c[X, Y]=-\omega(X, Y)
\end{aligned}
$$

$$
\omega=-d \alpha
$$


Since $\omega \in W^{2}(\varphi), \omega=0$, hence:

$$
H^{2}(\varphi)=0 .
$$

If $\mathbf{G}$ is semisimple, it can be written as the direct sum of its simple ideals. The adjoint representation is the direct sum of the adjoint representations of its simple ideals. It is readily verified that the cohomology groups similarly split up into a direct sum of the cohomology groups of the simple ideals. Finally, then, we have proved:

Theorem 3.8. If $G$ is a semisimple (finite dimensional) Lie algebra and if $\varphi$ is the adjoint representation of $\mathrm{G}$, then $H^{2}(\varphi)=0$.

We shall see in the next Section that this has an important consequence for the possibility of deformations of Lie algebra.

As we have seen, the existence of the subspaces $W^{r}(\varphi)$ is crucial to the above arguments. If everything is finite dimensional, and if $\mathbf{G}$ is semisimple, its existence can be deduced from the complete reducibility theorem for finite dimensional Lie algebras. In the original KodairaSpencer theory, its existence is deduced using a "Laplacian" operator. We can see what is involved here in the following terms:

Suppose that $\Delta$ is a linear transformation

$$
C^{r}(\varphi) \rightarrow C^{r}(\varphi)
$$

which commutes with the action of $\mathbf{G}$, i.e.

$$
X \Delta(\omega)=\Delta X(\omega) \text { for } X \in \mathbf{G}, \omega \in C^{r}(\varphi) .
$$

The aim is to choose $\Delta$ so that its kernel, i.e. the set of $\omega \in Z^{r}(\varphi)$ such that

$$
\Delta(\omega)=0,
$$

can serve as $H^{r}$. In the Kodaira-Spencer theory, the elements of $C^{r}(\varphi)$ were realized as tensor fields on a compact manifold, and $\Delta$ could be taken as a certain second-order elliptic differential operator. In our more Lie algebraic approach, it is natural to consider $\Delta$ as one of the Casimir operators of $\mathbf{G}$, i.e. an element in the universal enveloping algebra which commutes with AdG. In fact, let us examine the second order Casimir operator.

Now,

$$
\left.X^{2}(\omega)=X(d(X\rfloor \omega)\right)=d(X \perp(\omega)) \text { for } X \in G .
$$

Suppose $X_{1}, \ldots, X_{n}$ is a basis for $\mathrm{G}$ that is orthonormal with respect to the Killing form. Then,

Thus,

$$
\Delta=\sum g_{j} X_{j}^{2}, \quad \text { with } g_{j}= \pm 1 \text { for } j=1, \ldots, n \text {. }
$$

i.e.

$$
\left.\left.\Delta(\omega)=\sum_{j} g_{j} d(X\rfloor X(\omega)\right)=d\left(\sum_{j} g_{j} X_{j}\right\rfloor X_{j}(\omega)\right)
$$

$$
\Delta\left(Z^{r}\right) \subset d C^{r-1}
$$


Thus, if $Z^{r}(\varphi)$ is a direct sum of the kernel of $\Delta$ and $\Delta\left(Z^{r}(\varphi)\right.$ ) (which one would expect if $\Delta$ had something like an "eigenvector expansion") one would expect the kernel of $\Delta$ to serve as $W^{r}$. For example, suppose that $\omega \in Z^{1}(\varphi)$, and $\Delta(\omega)=0$. Then, for $Y \in \mathbf{G}$,

$$
\Delta(\omega)(Y)=0=\varphi(Y)\left(\sum_{j} g_{j} X_{j} \perp X_{j}(\omega)\right) ;
$$

Suppose, for example, that $\varphi(\mathbf{G})$ has no invariant vectors. Then, the condition that $\Delta(\omega)=0$ is :

$$
\sum_{j} g_{i} \varphi\left(X_{i}\right)\left(\omega\left(X_{j}\right)\right)=0 \text {. }
$$

We shall now leave the general cohomology theory at this point in order to review the applications to deformations of Lie algebras and their representations.

\section{Analytic continuation of Lie algebra structure}

Let $\mathbf{G}$ be a vector space with a "given" Lie algebra structure $(X, Y) \rightarrow[X, Y]$. Consider an "analytic continuation" of this structure, i.e., for each value of $\lambda$, a Lie bracket operation $(X, Y) \rightarrow[X, Y]_{\lambda}$ is given on $G$, reducing to the given one for $\lambda=0$, satisfying the Jacobi identity:

$\left[X,[Y, Z]_{\lambda}\right]_{\lambda}=\left[[X, Y]_{\lambda}, Z\right]_{\lambda}+\left[Y,[X, Z]_{\lambda}\right]_{\lambda}$ for $X, Y, Z \in \mathbf{G}$.

For $X, Y \in \mathbf{G}$, define:

$$
\omega(X, Y)=\frac{d}{d \lambda}[X, Y]_{\lambda / \lambda=0} .
$$

Differentiating (4.1) gives the identity:

$$
\begin{aligned}
& \omega(X,[Y, Z])+[X, \omega(Y, Z)] \\
& =\omega([X, Y], Z)+[\omega(X, Y), Z]+\omega(Y,[X, Z])+ \\
& \quad+[Y, \omega(X, Z)] .
\end{aligned}
$$

Let us interpret $\omega$ as a 2-cochain. Let $\varphi$ be the adjoint representation of $\mathbf{G}$; for $X \in \mathbf{G}, \varphi(X)$ is the linear transformation $Y \rightarrow[X, Y]$ of $\mathbf{G}$ into itself. Then, $\omega$ can be interpreted as an element of $C^{2}(\varphi)$. It is readily checked that condition (4.2) just means that:

i.e. $\omega \in Z^{2}(\varphi)$.

$$
d \omega=0 \text {, }
$$

Let us look at the meaning of the condition:

$$
\omega \in d C^{1}(\varphi) \text {. }
$$

Suppose that the $\lambda$-th Lie algebra is isomorphic to the original one, i.e., for each $\lambda$ there is a linear transformation

$$
A_{\lambda}: \mathbf{G} \rightarrow \mathbf{G} \text {, with } A_{0}=\text { identity }
$$


such that

$$
[X, Y]_{\lambda}=A_{\lambda}^{-1}\left[A_{\lambda} X, A_{\lambda} Y\right] \text { for } X, Y \in \mathbf{G}
$$

Define:

$$
\theta(X)=\left.\frac{d}{d \lambda} A_{\lambda}(X)\right|_{\lambda=0} \text { for } X \in \mathbf{G} .
$$

Interpret $\theta$ as an element of $C^{1}(\varphi)$. Differentiating (4.3) gives:

i.e.

$$
\omega(X, Y)=-\theta([X, Y])+[\theta(X), Y]+[X, \theta(Y)]
$$

hence:

$$
d \theta=\omega
$$

The element of $H^{2}(\varphi)$ determined by $\omega$ is zero if 4.3 is satisfied.

The Kodaira-Spencer theory then interprets the element of $H^{2}(\varphi)$ as the "first obstruction" to the existence of a one-parameter family of isomorphisms between the $\lambda$-th and the original Lie algebra structure. For example, if $\mathbf{G}$ is finite dimensional and semisimple, we have seen (Theorem 3.8) that $H^{2}(\varphi)=0$. Then, the deformation theory (e.g. [3]) applies to give the existence of the $A_{\lambda}$ satisfying (4.3): Deformations of semisimple algebras are "trivial" for the Kodaira-Spencer type of theory. However, as we have seen these are not "trivial" for our purposes, since we are interested in what can happen as $\lambda \rightarrow \infty$. In fact, the general theory comes into play here also: Suppose, for example, that the Lie algebra $[$,$] is an analytic function of \frac{1}{\lambda}$ at $\lambda=\infty$. Then, it can be regarded as an analytic continuation of the Lie algebra structure $[,]_{\infty}$, we contributes to $H^{2}\left(\varphi_{\infty}\right)$, where $\varphi_{\infty}$ is the adjoint representation of this $\lambda=\infty$ Lie algebra.

For example, let us apply this remark to the Lie algebra G of $S L(2, R)$, generated by elements $X, Y, Z$, with

$$
[Z, X]=Y ;[Z, Y]=-X
$$

Consider $A_{\lambda}$ given by:

$$
[X, Y]=-Z \text {. }
$$

$$
\begin{gathered}
A_{\lambda} Z=Z ; A_{\lambda} X=X / \lambda ; A_{\lambda} Y=Y / \lambda \\
{[Z, X]_{\lambda}=Y,[Z, Y]_{\lambda}=-X} \\
{[X, Y]_{\lambda}=-Z / \lambda .}
\end{gathered}
$$

et $\omega \in Z^{2}\left(\varphi_{\infty}\right)$ be the cocycle resulting from differentiating with respect to $1 / \lambda$ :

$$
\begin{gathered}
\omega(Z, X)=0=\omega(z, Y) . \\
\omega(X, Y)=-Z .
\end{gathered}
$$

Notice that the Lie algebra of $S L(2, R)$ can be reconstructed from the Lie algebra of $G_{\infty}$ and $\omega$. This fact will be generalized in Section 6 . 5 Commun. math. Phys., Vol. 3 


\section{Analytic continuation of Lie algebra homomorphisms}

Let $\mathbf{G}$ and $\mathbf{L}$ be Lie algebras. Let $\varphi: \mathbf{G} \rightarrow \mathbf{L}$ be a homomorphism from G to $\mathbf{L}$. A deformation or analytic continuation of $\varphi$ is a one-parameter family $\lambda \rightarrow \varphi_{\lambda}$ of homomorphisms reducing to the given one when $\lambda=0$.

Let $\varphi^{\prime}$ be the following representation of $\mathrm{G}$ by linear transformations on $L$ :

For $X \in \mathbf{G}, Y \in L, \varphi^{\prime}(X)(Y)=[\varphi(X), Y]$.

Define:

$$
\omega(X)=\left.\frac{d}{d \lambda} \varphi_{\lambda}(X)\right|_{\lambda=0} \text { for } X \in \mathbf{G} .
$$

Thus, $\omega \in C^{1}\left(\varphi^{\prime}\right)$. Let us differentiate the identity:

$$
\begin{aligned}
\varphi_{\lambda}([X, Y]) & =\left[\varphi_{\lambda} X, \varphi_{\lambda} Y\right] . \\
\omega([X, Y]) & =[\omega(X), \varphi(Y)]+[\varphi(X), \omega(Y)] \\
& =\varphi^{\prime}(X)(\omega(Y))-\varphi^{\prime}(Y)(\omega(X)) .
\end{aligned}
$$

It is equivalent to:

$$
d \omega=0 .
$$

Let us look for the condition that $\omega$ be a coboundary: Suppose that $\varphi_{\lambda}$ is obtained from $\varphi$ by changing it via an inner automorphism of $L$, i.e. there exists a curve $\lambda \rightarrow Y(\lambda)$ in $L$ such that:

$$
\begin{aligned}
\varphi_{\lambda} & =\operatorname{Ad} \operatorname{Exp}(Y(\lambda)) \varphi, \\
Y(0) & =0, \text { i.e. }
\end{aligned}
$$

Then,

$$
\varphi_{\lambda}(X)=\operatorname{Ad} \operatorname{Exp}(Y(\lambda))(\varphi(X)) \text { for } X \in \mathrm{G} .
$$

Put: $Z=\frac{d}{d \lambda} Y(\lambda)_{\lambda=0}$. Then,

hence:

$$
\omega(X)=[Z, \varphi(X)]=-\varphi^{\prime}(X)(Z),
$$

$$
\omega \in d C^{0}\left(\varphi^{\prime}\right) \text {. }
$$

Thus, we see that $H^{1}\left(\varphi^{\prime}\right)$ "obstructs" the possibility of writing the deformation $\lambda \rightarrow \varphi_{\lambda}$ in form (5.1).

For example, suppose that $\mathbf{L}$ consists of the set of all operators on a vector of space $V$, so that $\varphi$ defines a linear representation of $G$. Thus, analytic continuations of form (5.1) are "trivial" in the sense that each one is equivalent (by a change of basis, say) to the given one. For example, a Casimir operator of $\mathrm{G}$ will have the same value for all $\lambda$. Notice that if $\mathbf{G}$ is semisimple and if $\mathbf{L}$ is finite dimensional, $H^{2}\left(\varphi^{\prime}\right)=0$ : This is interpreted by saying that the finite dimensional representations of semisimple groups are "rigid", i.e. cannot be deformed in a non-trivial way. In fact, we know much more, from Lie algebra theory, namely that the equivalence classes of finite dimensional irreducible representations of a 
semisimple group form a discrete set, namely a lattice in its Cartan subalgebra determined by the maximal weights. However, notice how much simpler it is to prove the weaker rigidity theorem: this gives us hope that cohomology techniques at least might be useful in obtaining qualitative information about the structure of the set of unitary equivalence classes of unitary representations of non-compact semisimple Lie groups.

Let us investigate more precisely how the cohomology is related to the values of a Casimir operator of $G$. Suppose $\lambda \rightarrow \varphi_{\lambda}$ is a deformation of homomorphisms: $\mathbf{G} \rightarrow \mathbf{L}$, as before, and suppose

$$
\Delta=g_{j} X_{j}^{2}
$$

is a second order element of the universal enveloping algebra that lies in the center, i.e. is annihilated by $\operatorname{Ad} G$. Suppose that $\varphi(\Delta)$ lies in the center of the enveloping algebra of $\mathbf{L}^{3}$. $\left(X_{1}, \ldots, X_{n}\right.$ are elements of $G$; the indices $i, j, \ldots$ run between 1 and $n$, and we use the summation convention, $g_{1}, \ldots, g_{n}$ are scalars.)

Then,

$$
\left.\frac{d}{d \lambda} \varphi_{\lambda}(\Delta)\right|_{\lambda=0}=g_{i}\left(\omega\left(X_{i}\right) \varphi\left(X_{i}\right)+\varphi\left(X_{i}\right) \omega(X)\right) .
$$

Suppose $\omega \in d C^{0}\left(\varphi^{\prime}\right)$, i.e.

Then,

$$
\omega=d Z, \text { for } Z \in C^{0}\left(\varphi^{\prime}\right)=\mathbf{L} .
$$

Then,

$$
\omega(X)=[\varphi(X), Z] \text { for } X \in G .
$$

$\left.\frac{d}{d \lambda} \varphi_{\lambda}(\Delta)\right|_{\lambda=0}=g_{i}\left(\left[\varphi\left(X_{i}\right), Z\right] \varphi\left(X_{i}\right)+\varphi\left(X_{i}\right)\left[\varphi\left(X_{i}, Z\right]\right)=[\varphi(\Delta), Z]=0\right.$.

Suppose now that these conditions are satisfied: Let us look at the condition that $\left.\frac{d^{2}}{d \lambda^{2}} \varphi_{\lambda}(\Delta)\right|_{\lambda=0}$ be zero

$\left.\frac{d^{2}}{d \lambda^{2}} \varphi_{\lambda}(\Delta)\right|_{\lambda=0}=g_{j}\left(\frac{d^{2}}{d \lambda^{2}} \varphi_{\lambda}\left(X_{j}\right) \varphi\left(X_{j}\right)+2 \omega\left(X_{j}\right) \omega\left(X_{j}\right)+\right.$

Put:

$$
\left.+\varphi\left(X_{j}\right) \frac{d^{2}}{d \lambda^{2}} \varphi_{\lambda}\left(X_{j}\right)\right)\left.\right|_{\lambda=0} .
$$

Then, $\omega_{2} \in C^{1}\left(\varphi^{\prime}\right)$.

$$
\omega_{2}(X)=\left.\frac{d^{2}}{d \lambda^{2}} \varphi_{\lambda}(X)\right|_{\lambda=0} \text { for } X \in \mathbb{G} .
$$

Let us differentiate the identity twice:

$$
\begin{gathered}
\varphi_{\lambda}([X, Y])=\left[\varphi_{\lambda}(X), \varphi_{\lambda}(Y)\right] \text { for } X, Y \in \mathbf{G}, \\
\omega_{2}([X, Y])=\left[\omega_{2}(X), \varphi(Y)\right]+\left[\varphi(X), \omega_{2}(Y)\right]+2[\omega(X), \omega(Y)], \\
d \omega_{2}(X, Y)=2[\omega(X), \omega(Y)] .
\end{gathered}
$$

${ }^{3}$ For example, if $\mathbf{L}$ is the Lie algebra of operators on a vector space $V$, and $\varphi$ arises from an irreducible representation of $G$ on $V$, then, by ScHUR's lemma, $\varphi(\Delta)$ will satisfy this condition.

5* Commun. math. Phys., Vol. 3 
Now, we have defined the Lie derivative $\omega \rightarrow X(\omega)$ of a cochain by an element of $\mathbf{G}$. However, the vector space $\mathbf{L}$ in which cochains take their values is also a Lie algebra: We can define the Lie derivative $\omega \rightarrow Z(\omega)$ by an element $Z \in \mathbf{L}$ by the formula:

$$
Z(\omega)(X)=[Z, \omega(X)]
$$

(There is a special case of a construction investigated by NIJENHUIS and Richardson [3]: The cochain themselves can be made onto a Lie algebra.)

However, this Lie derivative does not commute with exterior differentiation:

$$
\begin{aligned}
d Z(\omega)(X, Y)= & {[\varphi(X), Z(\omega)(Y)]-} \\
& -[\varphi(Y), Z(\omega)(X)]-Z(\omega)([X, Y]) \\
= & {[\varphi(X),[Z, \omega(Y)]]-[\varphi(Y),[Z, \omega(X)]]-} \\
& -[Z, \omega([X, Y])] \\
= & {[Z,[\varphi(X), \omega(Y)]]-[[Z, \varphi(X)], \omega(Y)]-} \\
& -[z,[\varphi(Y), \omega(X)]]+[[Z, \varphi(Y)], \omega(X)]- \\
& -[Z, \omega([X, Y])] \\
= & Z(d \omega)(X, Y)-[[Z, \varphi(X)], \omega(Y)]+[[Z, \varphi(Y)], \omega(X)] .
\end{aligned}
$$

In our case, $d \omega=0, \omega(X)=[\varphi(X), Z]$. Hence,

Thus,

$$
d Z(\omega)(X, Y)=2[\omega(X), \omega(Y)] \text {. }
$$

i.e.

$$
d\left(\omega_{2}-Z(\omega)\right)=0
$$

$$
\omega_{2}-z(\omega) \in Z^{1}\left(\varphi^{\prime}\right)
$$

Let us again suppose its cohomology class is zero, i.e.

Then,

$$
\omega_{2}-Z(\omega)=d Z_{1}, \quad \text { with } \quad Z_{1} \in \mathbf{L} \text {. }
$$

$$
\begin{aligned}
& \left.\frac{d^{2}}{d \lambda^{2}} \varphi_{\lambda}(\Delta)\right|_{\lambda=0}=g_{i}\left(\omega_{2}\left(X_{1}\right) \varphi\left(X_{j}\right)+2 \omega\left(X_{j}\right) \omega\left(X_{j}\right)+\varphi\left(X_{i}\right) \omega_{2}\left(X_{j}\right)\right) \\
& =g_{i}\left(\left(\left[Z, \omega\left(X_{j}\right)\right]+\left[\varphi\left(X_{j}\right) Z_{1}\right)\right]\right) \varphi\left(X_{i}\right)+2 \omega\left(X_{j}\right) \omega\left(X_{j}\right)+ \\
& \quad+\varphi\left(X_{j}\right)\left(\left[Z, \omega\left(X_{j}\right)\right]+\left[\varphi\left(X_{j}\right), Z_{j}\right]\right) \\
& =g_{j}\left(\left[Z,\left[\varphi\left(X_{j}\right), Z\right]+\left[\varphi\left(X_{j}\right), Z_{1}\right]\right) \varphi\left(X_{j}\right)+\right. \\
& \quad+2\left[\varphi\left(X_{j}\right), Z\right]\left[\varphi\left(X_{j}\right), Z\right]+\varphi\left(X_{j}\right)\left(\left[Z,\left[\varphi\left(X_{j}\right), Z\right]+\varphi\left(X_{j}\right), Z_{j}\right]\right) \\
& =-[Z,[Z, \Delta]]+\left[\Delta, Z_{1}\right] .
\end{aligned}
$$

(Notice that this formula is general, and does not depend on $\Delta$ being a Casimir operator of $\mathbf{G}$, and holds no matter what the degree of $\Delta$. The method can be extended to the higher derivatives with respect to $\lambda$. We have then proved the following result: 
Theorem 5.1. Suppose $\varphi$ is representation of $\mathrm{G}$ by operators on a vector space in which the Casimir operators of $\mathbf{G}$ are multiples of the identity. Suppose $H^{1}\left(\varphi^{\prime}\right)=0$. Let $\lambda \rightarrow \varphi_{\lambda}$ be any analytic continuation of $\varphi$. Then, the values of the Casimir operators are independent of $\lambda$.

In particular, this theorem is useful in physics in interpreting the idea of analytic continuation of spin or angular momentum. For, this is just defined as an eigenvalue of the Casimir operator of $S O(3)$ in a representation: the theorem then asserts that to continue analytically a given representation of the Lie algebra in such a way that the value of the Casimir operator changes, it is necessary to continue into an infinite dimensional space.

\section{Cohomology of semidirect products}

Let $\mathbf{G}$ be a Lie algebra, and let $\varphi$ be the adjoint representation of $\mathbf{G}$. We have seen that, if $\mathbf{G}$ is semi-simple, the first and second cohomology groups relative to $\varphi$ are easy to compute. Basically, this is because all finite dimensional linear representations of $\mathrm{G}$ are completely reducible. The next simplest situation occurs when $\mathbf{G}$ has a subalgebra $\mathbf{K}$ such that the representation of $\mathbf{G}$ on the cohomology groups restricted to $\mathbf{K}$ is completely reducible. In this section, we shall present some typical work in this direction, without aiming for a complete analysis.

Rather we shall always aim at an understanding of the Poincaré group. Suppose that:

$$
H^{0}(\varphi)=0 \text {, i.e. G has no center }
$$

$Z^{1}(\varphi)=d C^{0}(\varphi)+W^{1}(\varphi)$, with $X\left(\omega^{1}(\varphi)\right) W^{1}(\varphi)$ for $X \in \mathbf{K}$.

As we have seen, (6.2) implies that:

hence :

$$
X(\omega)=0 \quad \text { for } \quad \omega \in W^{1}(\varphi), \quad X \in \mathbf{K},
$$

By (6.1) we have:

$$
d(X\lrcorner \omega)=0 .
$$

Also, for $X \in \mathbf{K}, Y \in \mathbf{G}$,

$$
\omega(\mathbf{K})=0 .
$$

$$
\begin{aligned}
0=d \omega(X, Y) & =[X, \omega(Y) \lambda-[Y, \omega(X) \lambda-\omega([X, Y]) \\
& =[X, \omega(Y)]-\omega([X, Y]) .
\end{aligned}
$$

Suppose that $\mathbf{P}$ is a subspace of $\mathbf{G}$ such that:

$$
\mathbf{G}=\mathbf{K}+\mathbf{P},[\mathbf{K}, \mathbf{P}] \subset \mathbf{P} .
$$

Then, we have:

Theorem 6.1. In addition to these assumptions, suppose that $\operatorname{Ad} \mathbf{K}$ acting in $\mathbf{P}$ is irreducible over the complex numbers and that $\mathbf{A d} \mathbf{K}$ acting on $\mathbf{K}$ contains no representation equivalent to $\mathrm{Ad} \mathbf{K}$ acting on $\mathbf{P}$, and that 
$[\mathbf{P}, \mathbf{P}]=0$. Then,

$$
\operatorname{dim} H^{1}(\varphi)=1 .
$$

Proof. Notice that the hypotheses that Ad $K$ acting in $P$ irreducible over the complex numbers means that any operator on $P$ commuting with Ad $K$ must be a multiple of the identity. Now, (6.4) implies that $\omega$, as a map $\mathbf{P} \rightarrow \mathbf{G}$, commutes with the action of AdK. Our assumption that $\mathbf{A d} \mathbf{K}$ acting on $\mathbf{K}$ contains no representation like AdK acting on $\mathbf{P}$ implies that:

Then, for $X, Y \in \mathbf{P}$,

$$
\omega(\mathbf{P}) \subset \mathbf{P}
$$

$$
\begin{aligned}
0=d \omega(X, Y)= & {[X, \omega(Y)]-[Y, \omega(X)]-\omega([X, Y]) } \\
= & a[X, Y]-a[Y, X] \\
& 2 a[X, Y] .
\end{aligned}
$$

This proves that $W^{1}(\varphi)$, hence also $H^{1}(\varphi)$, is spanned by the map $\mathbf{G} \rightarrow \mathbf{G}$ which is zero on $K$ and the identity on $\mathbf{P}$.

Remark: Suppose we do not assume that $[\mathbf{P}, \mathbf{P}]=0$. Then for $X, Y \in \mathbf{P}$,

$0=d \omega(X, Y)=2 a[X, Y]-a \quad$ (projection of $[X, Y]$ on $P$ ) .

Then, if $a \neq 0$, we must have $[\mathbf{P}, \mathbf{P}]=0$. For, otherwise,

$$
[\mathbf{P}, \mathbf{P}] \subset \mathbf{P} \text {. }
$$

(6.7) then implies that $[\mathbf{P}, \mathbf{P}]=0$. Thus, we have:

$$
H^{2}(\varphi)=0 \quad \text { if } \quad[\mathbf{P}, \mathbf{P}] \neq 0 .
$$

Now, let us turn to $H^{2}(\varphi)$. Suppose that a subspace $W^{2}(\varphi)$ exists with:

$$
\begin{gathered}
Z^{2}(\varphi)=W^{2}(\varphi) \oplus d C^{1}(\varphi), \\
X\left(W^{2}(\varphi)\right) \subset W^{2}(\varphi) \text { for } \quad X \in \mathbf{K} .
\end{gathered}
$$

Then, for $\omega \in W^{2}(\varphi), X \in \mathbf{K}$,

Suppose then that

$$
0=d(X\rfloor \omega)
$$

where

$$
X\rfloor \omega=\theta_{X}+d\left(Y_{X}\right),
$$

$$
\theta_{X} \in W^{\mathbf{1}}(\varphi), Y_{X} \in G \text {. }
$$

Then, $X \rightarrow \theta_{X}$ and $X \rightarrow Y_{X}$ define linear functions of $X$. (Here we must use condition (6.1)). Now, for $Z \in \mathbf{K}$,

$$
\begin{aligned}
Z(X\rfloor \omega) & =[Z, X]\lrcorner \omega \\
& =Z\left(\theta_{X}\right)+d\left[Z, Y_{X}\right] .
\end{aligned}
$$


But, $[Z, X]\lrcorner \omega=\theta_{[Z, X]}+d Y_{[Z, X]}$, i.e.

$$
\begin{aligned}
\theta=Z\left(\theta_{X}\right) & =\theta_{[Z, X]} \text { for } X, Z \in \mathbf{K} \\
Y_{[Z, X]} & =\left[Z, Y_{X}\right] \text { for } Z \in \mathbf{K} .
\end{aligned}
$$

Thus, the mapping $X \rightarrow Y_{X}$ commutes with the action of $\mathbf{K}$ on $\mathbf{G}$. Define $\alpha \in C^{1}(\varphi)$ as follows:

$$
\begin{array}{lll}
\alpha(X)=Y_{X} & \text { for } & X \in \mathbf{K} \\
\alpha(X)=0 & \text { for } & X \in \mathbf{P} .
\end{array}
$$

Then,

$$
d \alpha(X, Z)=[X, \alpha(Z)]-[Z, \alpha(X)]-\alpha([X, Z]) .
$$

Case 1: $Z \in \mathbf{K}$

$$
\begin{aligned}
d \alpha(X, Z)=(X \perp d \alpha(Z) & =\left[X, Y_{Z}\right]-\left[Z, Y_{X}\right]-Y_{[X, Y]} \\
& =-\left[Z, Y_{X}\right]=-d Y_{X}(Z) .
\end{aligned}
$$

Case 2: $Z \in \mathbf{P}$.

$$
d \alpha(X, Z)=-\left[Z, Y_{X}\right]-\alpha([X, Z])=-d Y_{X}(Z) .
$$

(We are assuming that $[\mathbf{K}, \mathbf{P}] \subset \mathbf{P}$ ).

In both cases we have:

In particular,

$$
X\rfloor d \alpha=-d Y_{X}
$$

$$
X\rfloor(\omega+d \alpha)=\theta_{X} \text { for } \quad X \in \mathbf{K} .
$$

Theorem 6.2. Suppose:

a) $\mathbf{K}$ acting as $Z^{1}(\varphi)$ and $Z^{2}(\varphi)$ is completely reducible.

b) $[\mathbf{K}, \mathbf{K}]=\mathbf{K}$.

Then, each cohomology class $H^{2}(\varphi)$ contains a cocycle $\omega$ such that

$$
X\rfloor \omega=0 \text { for all } X \in \mathbf{K} \text {. }
$$

Proof. Notice that hypothesis b) and (6.9) imply that:

$$
\theta_{X}=0 \text { for all } X \in K \text {. }
$$

(6.11) then completes the proof.

Through condition (6.12) we have succeeded in partially normalizing the elements of $W^{2}(\varphi)$. (Our goal is, of course, to compute $H^{2}(\varphi)$, at least in sufficiently simple, but interesting, cases.)

Suppose that $\omega \in Z^{2}(\varphi)$ satisfies (6.12). Then, also

$$
X(\omega)=0 \text { for all } X \in \mathbf{K} \text {. }
$$

Thus, the mapping $(X, Y) \rightarrow \omega(X, Y)$ of $\mathbf{P} \times \mathbf{P} \rightarrow \mathbf{G}$ commutes with the action of $\mathbf{K}$. We want to find a condition that

$$
\omega(\mathbf{P}, \mathbf{P}) \subset \mathbf{K} .
$$


Define $s: \mathbf{G} \rightarrow \mathbf{G}$ by the formula:

$$
\begin{array}{lll}
s(X)=X & \text { for } & X \in \mathbf{K} \\
s(X)=-X & \text { for } & X \in \mathbf{P} .
\end{array}
$$

Define the transform of $\omega$ by $s$, denoted by $s(\omega)$, as follows:

$$
s(\omega)(X, Y)=s(\omega(s X, s Y)) .
$$

(Notice that $s=s^{-1}$ ).

Lemma 6.3. If $s(\omega)=\omega$, then (6.13) is satisfied:

Proof. For $X, Y \in P$,

$$
\begin{aligned}
\omega(X, Y)=s(\omega)(X, Y) & =s(\omega(-X,-Y)) \\
& =s \omega(X, Y),
\end{aligned}
$$

i.e. $\omega(X, Y) \in \mathbf{K}$.

Now, we can put the pieces together:

Theorem 6.4. Let $\mathbf{G}_{c}=\mathbf{G}+i \mathbf{G}$ be the complexification of $\mathbf{G}$. Suppose $\mathbf{G}=\mathbf{K}+\mathbf{P}$, with $[\mathbf{K}, \mathbf{P}] \subset \mathbf{P},[\mathbf{P}, \mathbf{P}]=0$,

$$
[\mathbf{K}, \mathbf{K}] \subset \mathbf{K} \text {. }
$$

Let $s: \mathbf{G} \rightarrow \mathbf{G}$ be defined by (6.14). Extend $s$ to a complex linear transformation: $\mathbf{G}_{\boldsymbol{c}} \rightarrow \mathbf{G}_{c}$. Suppose that $\mathbf{K}$ is semisimple, and that the connected group of inner automorphisms of $\mathbf{G}_{c}$ generated by $K_{0}$ includes the transformation $s$. Then if $\omega \in Z^{2}(\varphi)$ satisfies (6.12), it also satisfies (6.13). In this case, we can define a one-parameter family of Lie algebra structures on the vector space $\mathrm{G}$ by the following formula:

$$
[X, Y]_{\lambda}=[X, Y]+\frac{1}{\lambda} \omega(X, Y) \text { for } X, Y \in \mathbf{G} .
$$

As $\lambda \rightarrow \infty$, this Lie algebra pass over to the Lie algebra on $\mathbf{G}$ with which we began.

Finally, if there is, up to a scalar multiple, just one skew-symmetric mapping $P \times P \rightarrow \mathbf{K}$ commuting with the action of $K$ on $K$ and $\mathbf{P}$, then

$$
\operatorname{dim} H^{2}(\varphi)=1 .
$$

If there are no such mappings (other than the one which maps onto zero), then

$$
\operatorname{dim} H^{2}(\varphi)=0 .
$$

Proof. Our hypotheses guarantee that $s(\omega)=\omega$, hence (6.13) is satisfied. It only remains to show that the formula (6.15) satisfies the Jacobi identity:

Now,

$$
\begin{aligned}
& {\left[X,[Y, Z]_{\lambda}\right]_{\lambda}=\left[X,[Y, Z]_{\lambda}\right]+\frac{1}{\lambda} \omega\left(X,[Y, Z]_{\lambda}\right)} \\
& =[X,[Y, Z]]+\left[X, \frac{\omega(Y, Z)}{\lambda}\right]+\frac{1}{\lambda}\left(\omega(X,[Y, Z])+\frac{1}{\lambda} \omega(X, \omega(Y, Z))\right) .
\end{aligned}
$$


Since $\omega(\mathbf{G}, \mathbf{G}) \subset \mathbf{K}$, and $K\rfloor \omega=0$, this equals

$$
[X,[Y, Z]]+\frac{1}{\lambda}[X, \omega(Y, Z)]+\frac{1}{\lambda} \omega(X,[Y, Z]) \text {. }
$$

The Jacobi identity now requires that:

$$
\begin{aligned}
& {[X, \omega(Y, Z)]+\omega(X,[Y, Z])+[Z, \omega(X, Y)]+} \\
& \quad+\omega(Z,[X, Y])-[Y, \omega(X, Z)]+\omega(Y,[X, Z])=0 .
\end{aligned}
$$

Notice that this is just the condition:

which we are given. q.e.d.

$$
d \omega=0,
$$

Remarks: This new Lie algebra structure []$_{\lambda}$ has the property that $\mathbf{K}$ is a symmetric subalgebra of $\mathbf{G}_{\lambda}$. The classification of symmetric subalgebras thus enables one to effectively compute $H^{2}(\varphi)$. We will do this in more detail in another paper.

Theorem 6.4 applies in case $G$ is the complex Lorentz group $S O(4, C)$. It is readily seen that $\operatorname{dim} H^{2}(\varphi)=1$. The corresponding Lie algebra $G_{\lambda}$ is just $S O(5, C)$, the complex de-Sitter group: The result as $\lambda \rightarrow \infty$ is the well-known contraction of the de-Sitter group into the Poincaré group ${ }^{4}$. This leads us to ask: Is this essentially the only group which can contract into the Poincaré group? We will now deal with this question.

\section{A uniqueness theorem for limits of Lie algebras}

Let $\mathbf{G}_{\infty}$ be a given Lie algebra. We have said that $G_{\infty}$ is the limit of a one-parameter family $\lambda \rightarrow[X, Y]_{\lambda}$ of Lie algebra structures on the same vector space as $\mathbf{G}_{\infty}$

$$
\lim _{\lambda \rightarrow \infty}[X, Y]_{\lambda}=[X, Y]_{\infty} \text { for } X, Y \in \mathbf{G}_{\infty} .
$$

Now, one may ask: What are the possibilities of structure of the Lie algebras $\mathbf{G}_{\lambda}$ (at least if $\lambda$ is sufficiently large)? The work of the last section provides us one method for severely limiting what seems at first sight appears to be a bewildering multiplicity of possibilities.

Let us suppose that the dependence on $\lambda$ is at least differentiable at $\lambda=\infty$, i.e. $[X, Y]_{\lambda}$ admits a Taylor expansion of the form:

$$
[X, Y]_{\lambda}=[X, Y]_{\infty}+\frac{1}{\lambda} \omega(X, Y)+\frac{1}{\lambda^{2}} \omega_{2}\left(X, Y, \frac{1}{\lambda}\right) \quad \text { for } \quad X, Y \in \mathbf{G}_{\infty} .
$$

As we have seen, $\omega \in Z^{2}(\varphi)$, where $\varphi$ is the adjoint representation of the Lie algebra $\mathbf{G}_{\infty}$, here determines an element of $H^{2}(\varphi)$. Further, we can assume that $\omega$ is any specified cocycle within its cohomology class, since changing the cocycle by a coboundary merely amounts to subjecting

${ }_{4}^{4}$ Of course, at the real level both $S O(4,1)$ and $S O(3,2)$ contract to the deSitter group. 
the vector space to a linear transformation. In particular, suppose that the cohomology class contains a cocycle satisfying the following condition:

$$
\omega(X, Y)\rfloor \omega=0 \text { for } X, Y \in \mathbf{G}_{\infty} .
$$

(We have seen in the last section that certain possibilities of structure for $\mathrm{G}_{\infty}$ lead to this conclusion.) Then, as the computation we used in proving Theorem 6.4 shows the following formula defines a Lie algebra structure for every value of $\lambda$ :

i.e.

$$
[X, Y]_{\lambda}^{1}=[X, Y]_{\infty}+\frac{1}{\lambda} \omega(X, Y),
$$

$$
[X, Y]_{\lambda}=[X, Y]_{\lambda}^{1}+\frac{1}{\lambda^{2}} \omega_{2}\left(X, Y, \frac{1}{\lambda}\right) .
$$

Suppose that the Lie algebra $\mathbf{G}_{\lambda}^{\prime}$ is semisimple. (Again, the work of the last section suggests many cases where this can be proved.) Now, the Lie algebra $G_{\lambda}$ is a deformation of the algebra $G_{\lambda}^{\prime}$ : Since the latter is semisimple, the rigidity theorem for semisimple algebras [3] implies that $G_{\lambda}$ is isomorphic to $G_{\lambda}^{\prime}$. Now, all the $G_{\lambda}^{\prime \prime}$ s are isomorphic among themselves: We thus have at least a sketch of a method for proving that, given certain assumptions about $\mathbf{G}_{\infty}$, only a certain number of Lie algebras may appear among the various ways of exhibiting $\mathbf{G}_{\infty}$ as a limit. Carrying out the details of this method will be done in a later paper.

In particular, these remarks do apply to the case where $G_{\infty}$ is the Poincaré group: The conclusion seems to be that it is only possible to "approximate" it by the de-Sitter groups, if one takes the method of approximation to be that described above.

Note added in proof: $\mathrm{R}$. Richardson has developed a more powerful method for handling the problem considered in section 7. His work will appear later.

\section{References}

[1] Hermann, R.: Analytic continuation of group representations, I. Commun. Math. Phys. 2, 251-270 (1966).

[2] - Differential geometry and the calculus of variation, to appear. New York: Academic Press.

[3] NiJenhuis, A., and R. Richardson: Cohomology and deformations in graded Lie algebras. Bull. Am. Math. Soc. 72, 1-29 (1966). 